\title{
PRONORMAL SUBGROUPS AND ZEROS OF CHARACTERS
}

\author{
GABRIEL NAVARRO \\ (Communicated by Pham Huu Tiep)
}

ABSTRACT. We give a characterization of when a pronormal subgroup of a solvable group is normal by using character theory.

\section{INTRODUCTION}

There seems to be a certain connexion between non-zero values of characters and normality in finite groups. For instance, if $G$ is a finite group, then a nonvanishing $x \in G$ (that is, an element on which no irreducible character takes the value zero) lies in $\mathbf{F}(G)$ whenever the order of $x$ is coprime to 6 ([DNPST] $)$. Dolfi, Pacifici, Sanus and Spiga have proved in DPSS] that if all the elements of a Sylow $p$-subgroup $P \in \operatorname{Syl}_{p}(G)$ are non-vanishing, then $P \triangleleft G$. (Other related results can be found in $\mathrm{MT}$. or $[\mathrm{NT}$.)

In fact, we have very recently proved in $\left[\mathrm{MN}\right.$ ] that if $P \in \operatorname{Syl}_{p}(G)$, then $P \triangleleft G$ if and only if $\chi(x) \neq 0$ for all $x \in P$ and all irreducible constituents $\chi$ of the permutation character $\left(1_{P}\right)^{G}$. This is the starting point of this note: If $H \triangleleft G$, then it is clear that $\chi(h) \neq 0$ for all $h \in H$ and all irreducible constituents $\chi$ of $\left(1_{H}\right)^{G}$, but the converse is not in general true. Are there other types of subgroups $H$, aside from Sylow subgroups, for which the converse holds?

Recall that a subgroup $H$ of $G$ is pronormal in $G$ if for every $g \in G$ we have that $H$ and $H^{g}$ are conjugate in $\left\langle H, H^{g}\right\rangle$. For instance, Sylow subgroups and Sylow normalizers are pronormal in every finite group, while Carter or Hall subgroups, among many others classes of subgroups, are pronormal in solvable groups.

If $H$ is a subgroup of $G$, let us denote by $\operatorname{Irr}\left(\left(1_{H}\right)^{G}\right)$ the set of the irreducible constituents of the permutation character $\left(1_{H}\right)^{G}$.

Theorem A. Suppose that $G$ is solvable and that $H$ is pronormal in $G$. Then $H \triangleleft G$ if and only if $\chi(h) \neq 0$ for all $\chi \in \operatorname{Irr}\left(\left(1_{H}\right)^{G}\right)$ and all $h \in H$.

Unfortunately, Theorem A is not true outside solvable groups: If $G=A_{7}$ and $H$ is any subgroup of $G$ isomorphic to $C_{2} \times C_{2}$, then it can be checked that $H$ is pronormal in $G$ and that $\chi(h) \neq 0$ for all $\chi \in \operatorname{Irr}\left(\left(1_{H}\right)^{G}\right)$ and all $h \in H$. As a matter of fact, we remark that any theorem implying the main result of $[\mathrm{MN}]$ or [DPSS] is not likely to have an elementary proof such as the one we give in the next section: all these results need the Classification of Finite Simple Groups.

Received by the editors September 3, 2012 and, in revised form, September 25, 2012.

2010 Mathematics Subject Classification. Primary 20C15.

Key words and phrases. Pronormal subgroups, zeros of characters.

This research was partially supported by the Spanish Ministerio de Educación y Ciencia, proyecto MTM2010-15296, and Prometeo/Generalitat Valenciana. 


\section{Proofs}

Proof of Theorem A. If $H \triangleleft G$ and $\chi \in \operatorname{Irr}(G)$ is an irreducible constituent of $\left(1_{H}\right)^{G}$, then $\chi_{H}=\chi(1) 1_{H}$, and therefore $\chi(h) \neq 0$ for all $h \in H$.

To prove the converse, we argue by induction on $|G|$. So we assume that $\chi(h) \neq 0$ for all $\chi \in \operatorname{Irr}\left(\left(1_{H}\right)^{G}\right)$ and all $h \in H$, and we prove that $H \triangleleft G$. We may assume that $H>1$. Let $N \triangleleft G$ and $g \in G$. Then $\left\langle H, H^{g}\right\rangle \subseteq\left\langle N H, N H^{g}\right\rangle$, and we see that $N H / N$ is pronormal in $G / N$. If $\chi \in \operatorname{Irr}(G)$ lies over $1_{H N}$, then $\chi$ lies over $1_{H}$, and therefore $\chi(h) \neq 0$ for all $h \in H$. If also $N \subseteq \operatorname{ker}(\chi)$, then $\chi(n h)=\chi(h) \neq 0$ for all $n \in N, h \in H$, and therefore $N H / N \triangleleft G / N$ if $N>1$ by the inductive hypothesis. Thus, if $1<N \triangleleft G$, then we have that $N H \triangleleft G$. In particular, we may assume that core $_{G}(H)=1$.

Now let $N$ be a minimal normal subgroup of $G$. Let $g \in G$. Since $N H \triangleleft G$, we have that $\left\langle H, H^{g}\right\rangle \subseteq N H$, and therefore $H^{g}=H^{n}$ for some $n \in N$. Thus $G=N \mathbf{N}_{G}(H)$. Since $N \cap H \triangleleft \mathbf{N}_{G}(H)$ and $N \cap H \triangleleft N$ because $N$ is abelian, then we deduce that $N \cap H \triangleleft G$. Thus $N \cap H=1$ because $H$ has a trivial core. Also $\mathbf{C}_{H}(N) \triangleleft \mathbf{N}_{G}(H)$, and since $\left[\mathbf{C}_{H}(N), N\right]=1$, we deduce that $\mathbf{C}_{H}(N)=1$, so that $H$ acts faithfully on $N$.

Now, let $K / N$ be a chief factor of $G$ where $K \subseteq N H$, so that $K / N$ is an abelian $p$-group for some prime $p$. Since the abelian $p$-group $K \cap H$ acts faithfully on the necessarily $p^{\prime}$-group $\operatorname{Irr}(N)$, we can conclude then that there is a regular orbit; that is, there exists $\lambda \in \operatorname{Irr}(N)$ such that no element of $K \cap H$ stabilizes $\lambda$. (See for instance Corollary 3.4 of $\left[\mathrm{IsB}\right.$.) Thus $\theta=\lambda^{K} \in \operatorname{Irr}(K)$ by the Clifford correspondence. Since

$$
\left(\left(1_{H}\right)^{N H}\right)_{N}=\left(1_{1}\right)^{N}=\rho_{N}
$$

is the regular character of $N$, it follows that some irreducible constituent $\mu \in$ $\operatorname{Irr}\left(\left(1_{H}\right)^{N H}\right)$ lies over $\lambda$. Hence, if $\chi \in \operatorname{Irr}(G)$ lies over $\mu$, then $\chi$ lies over $1_{H}$ and over $\lambda$. In particular, $\chi$ lies over $\theta=\lambda^{K}$. However, $\theta^{g}=\left(\lambda^{g}\right)^{K}$ vanishes on every $1 \neq x \in K \cap H$ for all $g \in G$. Hence so does $\chi$ by Clifford's theorem. This is a contradiction that concludes the proof of the theorem.

To end this note, we change our question: are there groups for which the nonvanishing and the normality conditions are equivalent? Even for nilpotent groups this is not a complete triviality.

(2.1) Theorem. Suppose that $G$ is nilpotent and let $H \leq G$. Then $H \triangleleft G$ if and only if $\chi(h) \neq 0$ for all $\chi \in \operatorname{Irr}\left(\left(1_{H}\right)^{G}\right)$ and all $h \in H$.

Proof. We assume that $\chi(h) \neq 0$ for all $\chi \in \operatorname{Irr}\left(\left(1_{H}\right)^{G}\right)$ and all $h \in H$, and we prove that $H \triangleleft G$. We assume that $H>1$. Let $Z$ be a minimal normal subgroup of $G$ so that $|Z|=p$ a prime and $Z$ is central. By induction, we have that $Z H \triangleleft G$. Thus we may assume that the core in $G$ of $H$ is trivial. Hence $Z \cap H=1$. Now, let $K / Z$ be a chief factor of $G$, where $K$ is contained in $Z H$. Thus $|K / Z|=q$, a prime. If $p \neq q$, then $K \cap H$ is a normal Sylow $q$-subgroup of $K$, so it is normal in $G$. But the core of $H$ is trivial, and this is not possible. So we may assume that $|K|=p^{2}$. Let $\langle h\rangle=H \cap K=Q$, which we know is not normal in $G$. Let $1 \neq \lambda \in \operatorname{Irr}(Z)$ and let $\theta=\lambda \times 1_{Q}$. Suppose that $g \in G$ does not centralize $h$. Since $(Z h)^{g}=Z h$, then we have that $h^{g}=z h$ for some $z \neq 1$. Now $h^{g^{i}}=z^{i} h$ for $i \geq 0$, and we see that the conjugacy class of $h$ in $G$ has size $p$. Hence $\left|G: \mathbf{C}_{G}(h)\right|=p$ and

$$
G=\mathbf{C}_{G}(h) \cup \mathbf{C}_{G}(h) g \cup \ldots \cup \mathbf{C}_{G}(h) g^{p-1}
$$


is a disjoint union. Also, since $Q=\operatorname{ker}(\theta)$, it easily follows that the stabilizer of $\theta$ in $G$ is $\mathbf{N}_{G}(Q)=\mathbf{C}_{G}(Q)=\mathbf{C}_{G}(h)$. Now, let $\chi \in \operatorname{Irr}(G)$ be over $\theta$. By Clifford's theorem, we have that

$$
\chi_{K}=e \sum_{j=0}^{p-1} \theta^{g^{j}}
$$

for some integer $e$. Then

$$
\chi(h)=e \sum_{j=0}^{p-1} \theta^{g^{j}}(h)=e\left(1+\lambda(z)+\cdots+\lambda\left(z^{p-1}\right)\right)=0
$$

because $\left[\lambda, 1_{Z}\right]=0$. This is a contradiction which proves the theorem.

If $G=C_{3} \times S_{3}$, then every subgroup contained in the Fitting subgroup $F$ of $G$ is non-vanishing, while there is a subgroup inside $F$ which is not normal in $G$. So Theorem (2.1) does not hold even for supersolvable groups.

Also, we would like to point out that we do not know of any solvable group $G$ having a subgroup $H$ such that $\chi(h) \neq 0$ for all $\chi \in \operatorname{Irr}\left(\left(1_{H}\right)^{G}\right)$ and all $h \in H$, and where $H$ is not subnormal in $G$. This would imply Theorem A and certainly be related to the main open problem in [NW].

\section{ACKNOWLEDGEMENTS}

The author thanks M. Isaacs and Tom Wolf for helpful conversations on this subject.

\section{REFERENCES}

[DNPST] Silvio Dolfi, Gabriel Navarro, Emanuele Pacifici, Lucia Sanus, and Pham Huu Tiep, Non-vanishing elements of finite groups, J. Algebra 323 (2010), no. 2, 540-545, DOI 10.1016/j.jalgebra.2009.08.014. MR2564856(2010k:20015)

[DPSS] Silvio Dolfi, Emanuele Pacifici, Lucia Sanus, and Pablo Spiga, On the orders of zeros of irreducible characters, J. Algebra 321 (2009), no. 1, 345-352, DOI 10.1016/j.jalgebra.2008.10.004. MR2469367(2009k:20013)

[Is] I. Martin Isaacs, Character theory of finite groups, AMS Chelsea Publishing, Providence, RI, 2006. Corrected reprint of the 1976 original [Academic Press, New York; MR0460423]. MR2270898

[IsB] I. Martin Isaacs, Finite group theory, Graduate Studies in Mathematics, vol. 92, American Mathematical Society, Providence, RI, 2008. MR2426855 (2009e:20029)

[INW] I. M. Isaacs, Gabriel Navarro, and Thomas R. Wolf, Finite group elements where no irreducible character vanishes, J. Algebra 222 (1999), no. 2, 413-423, DOI 10.1006/jabr.1999.8007. MR.1733678 (2000k:20008)

[MN] Gunter Malle and Gabriel Navarro, Characterizing normal Sylow p-subgroups by character degrees, J. Algebra 370 (2012), 402-406, DOI 10.1016/j.jalgebra.2012.07.050. MR:2966846

[MT] S. Marinelli and P. H. Tiep, Zeros of real irreducible characters of finite groups, J. Algebra and Number Theory, to appear.

[NT] Gabriel Navarro and Pham Huu Tiep, Degrees and p-rational characters, Bull. Lond. Math. Soc. 44 (2012), no. 6, 1246-1250, DOI 10.1112/blms/bds054. MR3007657

Departament d’Àlgebra, Universitat de València, 46100 Burjassot, València, Spain

E-mail address: gabriel@uv.es 